\title{
Research on the Allocation and Management of Prehospital Emergency Medical Devices in Beijing Scenic Areas
}

\author{
Honglin Yang, Yadong Wang*, Bo Zheng \\ School of Health Administration and Education, Capital Medical University, Beijing, China \\ Email address: \\ 407457292@qq.com(Honglin Yang), yadong61@ccmu.deu.cn(Yadong Wang), zhengbo1018@163.com(Bo Zheng) \\ ${ }^{*}$ Corresponding author
}

To cite this article:

Honglin Yang, Yadong Wang, Bo Zheng. Research on the Allocation and Management of Prehospital Emergency Medical Devices in Beijing Scenic Areas. Clinical Medicine Research. Vol. 7, No. 2, 2018, pp. 43-50. doi: 10.11648/j.cmr.20180702.12

Received: April 28, 2018; Accepted: May 24, 2018; Published: June 11, 2018

\begin{abstract}
The objective of this work is to understand the present situation of prehospital emergency work in Beijing scenic areas, including the status and conditions of first-aid equipment configurations, and an analysis of existing problems. The purpose of this research is to advance configuration suggestions and management methods suitable for scenic areas. Methods: A questionnaire was used to investigate the status of first-aid medical equipment in the $3 \mathrm{~A}-5 \mathrm{~A}$ scenic areas in Beijing. In assessing the appropriate configuration of prehospital first aid contents and forms at these scenic areas, the experts of the rescue team and the relevant staff of the Tourism Bureau were interviewed on the disposition and daily management of the medical equipment. Results: The content of first-aid medical equipment in various scenic areas is uneven. Much of the medical equipment is too outdated, and the level of first-aid service needs to be improved. Experts believe that the current configuration involves more clinical medication than equipment and often contains a combination of emergency equipment commonly used in hospitals. The key aspects of management should include personnel and training, maintenance and updating. Conclusion: The first aid configuration of the scenic areas is classified and managed in the form of a first aid package, which should mainly include basic medical equipment that the general public can use and equipment that can be used by some professional first aid personnel. The Health Bureau needs to make clear the training and maintenance of personnel and update the division of labor in order to ensure the normal operation of prehospital care. In addition, it is suggested that an automated external defibrillator (AED) should be deployed in favored locations with high traffic volumes so that patients with cardiac arrest can be rescued in time.
\end{abstract}

Keywords: Prehospital Emergency, Emergency Equipment, Public Places, Emergency Management

\section{Introduction}

In recent years, with the enhancement of first aid awareness and the increased variety of public places in China, people have begun to pay more attention to first aid work in public places and put forward higher requirements for the development of prehospital first aid. The regulations on the administration of hygiene in public places, formulated by the State Council, have clearly defined the scope of public places and their emergency treatment obligations [1]. In addition, the Beijing Pre-Hospital Emergency Service Ordinance also requires that schools, railway stations, sports venues, scenic areas and other public places be equipped with corresponding emergency facilities and medicines. Because of the many types of public spaces, their large human flows and complex environments, it is difficult to implement rescues once emergencies arise. It is necessary to standardize the medical equipment and daily management in order to ensure the smooth implementation of first aid. The prehospital first aid work in public areas with concentrated populations is not only an important context for evaluating the level and ability of a city's first aid work but also an important part of the construction of the emergency medical system [2].

Therefore, this study, focusing on scenic locations as an example, uses a questionnaire survey and personal interviews to understand the status of emergency medical equipment allocation in Beijing scenic areas, analyzes the existing problems with the aid of expert consultation, and advances some suggestions based on two aspects of the configuration content and management methods in order to unify the configuration and correlation of emergency medical 
equipment in scenic areas. This study provides guidance and assistance for the formulation of norms and provides protection for emergency patients in scenic areas.

\section{Materials and methods}

\subsection{Literature Research}

The researchers used "scenic area first aid", "prehospital first aid" and "prehospital first aid in public places" as the keywords for literature retrieval. Then, the related management methods for emergency equipment in public places at various levels, including Beijing and other areas, were evaluated to figure out the common diseases and the distribution of existing emergency situations in various scenic areas. Standards and management methods from public places other than scenic areas provided reference material for compiling the questionnaire and interview outline. The management methods collected were summarized and integrated, and word frequency analysis was used to form a framework. At the same time, the researchers drew lessons from the relevant configuration programs that had been introduced at home and abroad to develop continuous integration and arrangements to form the management methods of first-aid drugs for the scenic areas.

\subsection{Personal In-Depth Interview}

The researchers interviewed staff from the Beijing Tourism Bureau and the relevant staff of scenic areas to understand the current regulations on the allocation of first-aid drugs, the status of the emergency facilities and the allocation of drugs, and the needs of the various public places. In addition, they were asked to express their opinions on the requirements for first aid in public places and their opinions on the regulations on prehospital medical emergency services in Beijing.

\subsection{Expert Consultation}

The clinicians, emergency management experts and representatives of the rescue team were invited to discuss the configuration and specific content of emergency medical equipment in public places. Experts reviewed the problems in the first aid configuration and management at the scenic areas and advanced solutions. The experts invited include first aid specialists and emergency rescue experts from 120 emergency centers, Xuanwu hospitals, and volunteer rescue teams.

\subsection{The Questionnaire Survey}

In this study, staff from all of the 3A-5A level scenic areas in Beijing were selected as the survey subjects, and the questionnaires were filled out in the form of network questionnaires. A total of 51 questionnaires were received. The contents of the investigation mainly referred to the "the standard of Red Cross First Aid Kit (M281745)", " Railway Red Cross medicine box configuration directory (2015)", and related technical data and teaching materials [3, 4], including the understanding of first aid work, the emergency department and setting personnel, and the allocation of AED and the specific configuration of first-aid medical equipment.

\section{Results}

\subsection{Research Results}

The research team found Taiwan's " Management measures for necessary emergency equipment in public places" [5], Manchester City University's "First Aid Handbook" [6] and 3 domestic and foreign first-aid equipment management guidance documents [7-9], and referenced "Kunming Wujiaba Airport medical emergency rescue equipment management experience" [10] and "emergency management science" [11], a book on the management of first-aid equipment. The researchers read and focused on all of the above information and then used Atlas. ti software to calculate word frequency statistics (Table 1).

Table 1. Key words analysis results.

\begin{tabular}{llll}
\hline Content & Frequency & Content & Frequency \\
\hline AED & 10 & Professional management & 2 \\
Maintenance \& update & 8 & Encouragement policy & 4 \\
Record & 5 & Storage & 2 \\
Staff training & 5 & Guide card & 1 \\
Personnel identification & 5 & Qualification retraining & 2 \\
Responsibilities & 4 & Training institutions & 2 \\
Waste treatment & 4 & Use management & 3 \\
Equipment positioning & 2 & Supervision & 2 \\
\hline
\end{tabular}

With regard to word frequency, the Top 5 are AED related terms, equipment maintenance updates, usage records, personnel training, personnel identification and contact. This phenomenon fully shows that the emphasis of the existing management measures lies in the effective management of AED in public places. The main contents of various management methods are fully explained, especially for the management of AED. At present, our country's AED deployment is just starting, and the usage, configuration, daily management and other aspects of this equipment all need to be gradually standardized. Therefore, the formulation of specific items should be focused on in the corresponding framework, and these five key words should be relatively detailed and standardized. The other keywords should also be involved in the corresponding framework. According to the study of the specific data content and the result of the word frequency 
analysis, the research group summarizes and forms the preliminary framework of the management method and the corresponding specific content, as shown in Figure 1.

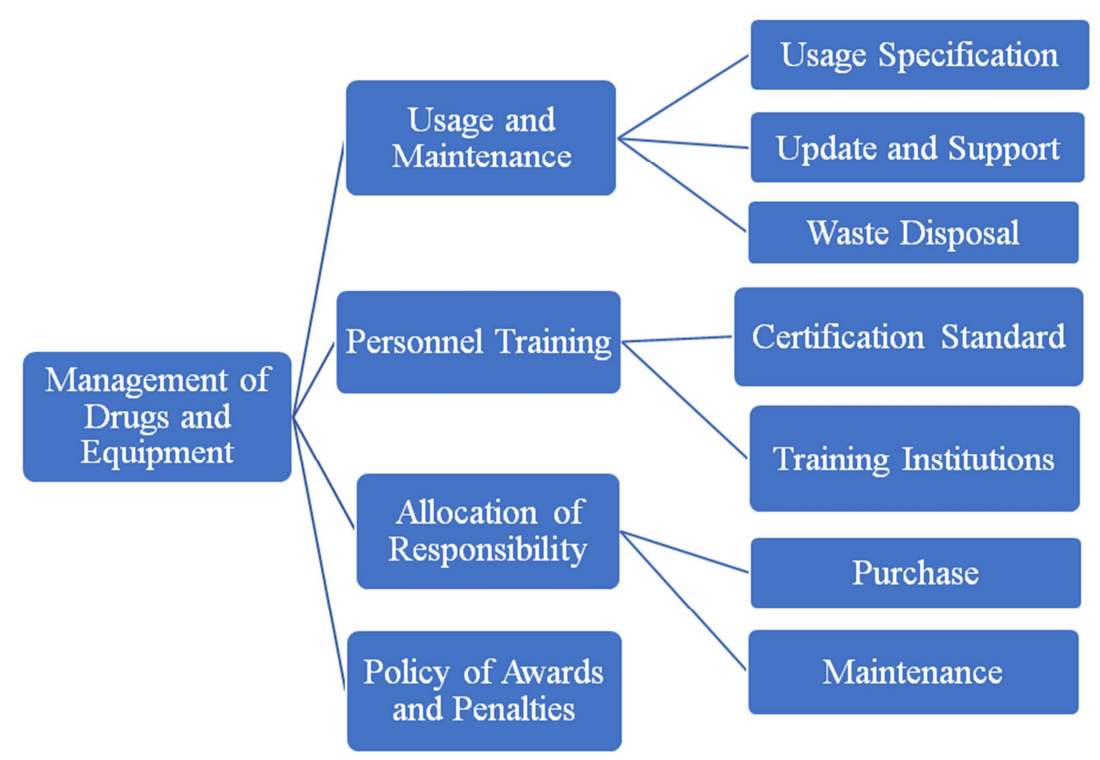

Figure 1. Framework for management.

\subsection{Personal Interview Results}

Regarding the configuration of equipment, the relevant staff of the Municipal Tourism Bureau said that the state's document, "the division and evaluation of the quality grade of tourist attractions," stipulates that the scenic areas are divided into 5 levels, 1A-5A, of which 5A, 4A and 3A scenic areas are required to establish emergency rescue mechanisms, set up medical rooms and are to be equipped with full-time medical personnel. The framework for the basic emergency work flow is quite complete. The $2 \mathrm{~A}$ and $1 \mathrm{~A}$ scenic areas are only required to be equipped with essential medicines and to handle accidents properly. The main reason for this difference is that the income at the $2 \mathrm{~A}$ and $1 \mathrm{~A}$ scenic areas is weak, the passenger flow is low, and there is no ability to undertake high-level first aid services. The staff also suggested that the unified standard of emergency medical equipment allocation in scenic areas can help to standardize the management of first aid and effectively improve the efficiency of emergency work. The management department of scenic areas should reduce the requirements in the formulation of the content configuration and stress the basic emergency preparation. In addition, some scenic areas are equipped with corresponding first-aid equipment according to their special needs, such as emergency poles, rescue boxes, etc. In general, there is no unified provision in the specific configuration of emergency medical equipment. It is necessary to formulate relevant standard documents for the allocation of medical equipment. However, it needs to be clear that there are there are essential differences in prehospital first aid between scenic area and the hospital. The former should focus on saving the patients' lives and transfer them to the corresponding medical institutions in time. In regard to the management methods, the scenic area staff are more concerned about the responsibility of the purchase and maintenance of medical equipment. At present, the scenic areas are used to dealing with general problems. If unified medical equipment is used in the scenic area of the city, the scenic area staff will contact the 120 emergency vehicles in the city for additional the protection on the scene during the peak period of the holidays. In these situations, the questions of who rescues, using which criteria, and how to carry out first aid training must be clarified by the management method. Moreover, if the AED is configured, the relevant leaders of the scenic area also expressed doubts regarding responsibility if the rescue is a failure.

\subsection{Questionnaire Survey Results}

\subsubsection{Configuration of the First-Aid Kit in Scenic Areas}

The survey showed that 48 of the 51 surveyed scenic areas were equipped with first-aid boxes, which were in good condition. However, the specific content configuration is limited to conventional or even dated emergency equipment, such as blood pressure meters, flashlights, thermometers, trigonometric towels, conventional bandages and gauze, tourniquets and band aids; the ratio of repurposed equipment is often more than $50 \%$. The ratio of new and professional first-aid equipment is not high. For example, there are only 8 scenic areas (16.67\%) equipped with a spinning tourniquet, 6 places $(12.5 \%)$ equipped with a self-adhesive tourniquet, 4 places $(8.33 \%)$ equipped with a mask and 10 places $(20.83 \%)$ equipped with a fracture splint. Only 13 areas (27.08\%) have medical saline. Availability rates of auxiliary emergency rescue equipment, such as cardiopulmonary resuscitation (CPR) mask, heat insulation blanket, escape rope, whistle and other similar content, are below $20 \%$. In particular, fluorescent rods for the identification of acute patients have not been paid adequate attention. In the scenic areas with large passenger volume and complex environments, the fluorescent bar is an 
important prop for emergency calls and identification and can help the emergency personnel find the patient's position in time and protect the patient amid the crowd. At the same time, there are many scenic areas that cannot detail the first aid configuration of their own units, as detailed in Table 2 .

Table 2. Configuration of first aid kit contents in scenic areas.

\begin{tabular}{|c|c|c|c|}
\hline Content & Yes & No & Don`t know \\
\hline Blood pressure meter & $25(52.08 \%)$ & $8(16.67 \%)$ & $15(31.25 \%)$ \\
\hline thermometer & $36(75 \%)$ & $3(6.25 \%)$ & $9(18.75 \%)$ \\
\hline Pocket Flashlight & $24(50 \%)$ & $9(18.75 \%)$ & $15(31.25 \%)$ \\
\hline Waterproof band-aid & $44(91.67 \%)$ & $0(0 \%)$ & $4(8.33 \%)$ \\
\hline Ordinary gauze bandage & $34(70.83 \%)$ & $5(10.42 \%)$ & $9(18.75 \%)$ \\
\hline Medical elastic bandage & $21(43.75 \%)$ & $12(25 \%)$ & $15(31.25 \%)$ \\
\hline Triangle & $23(47.92 \%)$ & $8(16.67 \%)$ & $17(35.42 \%)$ \\
\hline Common tourniquet & $27(56.25 \%)$ & $7(14.58 \%)$ & $14(29.17 \%)$ \\
\hline Rubber tourniquet & $15(31.25 \%)$ & $14(29.17 \%)$ & $19(39.58 \%)$ \\
\hline Spinning tourniquet & $8(16.67 \%)$ & $19(39.58 \%)$ & $21(43.75 \%)$ \\
\hline Rinse with normal saline & $13(27.08 \%)$ & $14(29.17 \%)$ & $21(43.75 \%)$ \\
\hline Fracture splint & $10(20.83 \%)$ & $15(31.25 \%)$ & $23(47.92 \%)$ \\
\hline CPR Mask & $4(8.33 \%)$ & $20(41.67 \%)$ & $24(50 \%)$ \\
\hline Automatic fire source & $7(14.58 \%)$ & $16(33.33 \%)$ & $25(52.08 \%)$ \\
\hline Light stick & $1(2.08 \%)$ & $21(43.75 \%)$ & $26(54.17 \%)$ \\
\hline
\end{tabular}

The first aid kits for the scenic areas were mainly equipped with common passenger medicine, including 32 locations $(66.67 \%)$ with Iodophor, $29(60.42 \%)$ with cool oil, 32 (66.67\%) with Quick-Acting Heart Reliever, 35 (72.92\%) with Huoxiang Zhengqi Wan, and 31 (64.58\%) with Yunnan Baiyao. Other clinical drugs, such as those for allergies and asthma (Promethazine Hydrochloride tablets, Carbocisteine tablets, Diprophylline tablets, etc.) are present in less than $10 \%$ of kits. At present, the configuration can meet the emergency needs of patients with heat stroke, trauma and sudden myocardial infarction, but there is a lack of treatment for asthma and other sudden respiratory diseases. There are also many scenic areas that do not understand the allocation of drugs in their own unit, as detailed in Table 3.

Table 3. Configuration of first aid kit medications in scenic areas.

\begin{tabular}{llll}
\hline Content & Yes & No & Don`t know \\
\hline Iodophor & $32(66.67 \%)$ & $3(6.25 \%)$ & $13(27.08 \%)$ \\
Cool oil & $29(60.42 \%)$ & $5(10.42 \%)$ & $14(29.17 \%)$ \\
Quick-Acting Heart Reliever & $32(66.67 \%)$ & $8(16.67 \%)$ & $8(16.67 \%)$ \\
Huoxiang Zhengqi Wan & $35(72.92 \%)$ & $8(16.67 \%)$ & $5(10.42 \%)$ \\
Yunnan Baiyao & $31(64.58 \%)$ & $8(16.67 \%)$ & $9(18.75 \%)$ \\
Amika Huangmin Tablets & $10(20.83 \%)$ & $17(35.42 \%)$ & $21(43.75 \%)$ \\
Carbocisteine & $1(2.08 \%)$ & $19(39.58 \%)$ & $28(58.33 \%)$ \\
Pediatric Paracetamol & $4(8.33 \%)$ & $16(33.33 \%)$ & $28(58.33 \%)$ \\
Oral rehydration salt powder & $5(10.42 \%)$ & $18(37.5 \%)$ & $25(52.08 \%)$ \\
Promethazine hydrochloride tablets & $2(4.17 \%)$ & $18(37.5 \%)$ & $28(58.33 \%)$ \\
Pediatric fever stickers & $7(14.58 \%)$ & $16(33.33 \%)$ & $25(52.08 \%)$ \\
Diprophylline tablets & $5(10.42 \%)$ & $18(37.5 \%)$ & $25(52.08 \%)$ \\
\hline
\end{tabular}

\subsubsection{Medicine Disposition of the Infirmary (Emergency Station)}

In regard to the first aid clinical setting, 28 (54.9\%) scenic areas have set up medical rooms or first aid points. However, there are $23(45.1 \%)$ scenic areas that have no infirmary or emergency station. Of the 28 scenic areas that have established an infirmary, $14(50 \%)$ have stretchers, 16 (57.14\%) have a rescue bed, and other specialized rescue equipment (such as neck care, spinal fixed board, cardiopulmonary resuscitation bag, etc.) are available in only a few. None of the surveyed scenic areas are configured for sputum suction apparatus, multifunctional defibrillation/monitoring/pacemaker or respiratory balloon, indicating that the medical conditions in the emergency setting of the scenic area cannot reach the level of professional clinical hospital first aid. Therefore, the focus should be on the lifting and transfer of the patients. In the drug reserve, almost no scenic areas are equipped with clinical first aid drugs (such as adrenalin, dopamine, erect hemostat and equality of blood) because these clinical medications must be administered by a professional physician and are beyond the scope of first aid work in scenic areas. Additionally, there is a high cost to the equipment and drugs needed for professional hospital first aid. There are certain requirements for the rescue environment, which cause a gap in the first aid level between the infirmary of scenic areas and the emergency room of hospitals. 


\subsection{Results of Expert Consultation}

\subsubsection{Medical Equipment Content Configuration}

Experts and emergency rescue team volunteer representatives believe that the present status of emergency medical equipment allocation to Beijing scenic areas is at a more basic level, and the unified configuration standard should not be too high. The purpose of configuring first-aid drugs is to encourage and support public participation in prehospital first aid for emergency patients. When emergencies arise, the basic first-aid drugs are accessible. In addition, the focus of first aid in public places is to save the life of the patient, stabilize their vital signs, and wait for the arrival of professional medical staff for more technical procedures, including outside injury hemostasis, CPR and the use of an AED [11]. For the scenic areas, it is suggested that the first aid kit should be arranged in the form of a first aid package to meet the general emergency needs of trauma dressing, debridement and disinfection and so on, and should limit the involvement of clinical medical behavior as much as possible. The first aid package should be provided to the public or scenic spot's staff who have first-aid qualifications at the scene. To save the life of emergency patients, the Health Bureau should carry out basic and necessary maintenance measures and strive for reduced time to professional medical staff help for clinical first aid follow-up. The contents of first aid packages should be considered general and specific. In public places such as scenic areas, most common emergencies are trauma and cardiac disease [12-15]. Based on this consideration, in the actual configuration, the main items should be updated equipment and those items with a large number of uses, including dressings, bandages, train gelation, gauze, and ice bags. In addition, the movement of an injured patient after a fall may cause damage to the neck, so it is suggested that neck supports be included in the basic equipment [16, 17]. Drugs can only be directed against common heart diseases, such as myocardial infarction, and kits should be equipped with quick-acting heart relievers and other drugs.

In addition, most experts agree with the inclusion of AED in the configuration. The only fundamental and effective measure to prevent sudden death patients outside the hospital is to carry out CPR in time, but the rate of CPR-related knowledge and skills in the public of our country is very low [18]. Zhang et al., in their recent study [19], pointed out that only $4.27 \%$ of the first witnesses performed CPR outside the large and medium cities in China, Li Zonghao and others pointed out that the success rate of cardiopulmonary resuscitation was lower than $1 \%$ [20] in our country. The fact that these patients cannot get timely treatment for cardiac arrest is not due to a technical reason, but mainly due to the public's lack of awareness that prevents emergency measures in time, leading to delayed rescue time [21]. As large areas of human flow with complex environments, the possibility of sudden cardiac arrest in scenic areas is very high, especially in the peak season. The configuration of AED provides the patient with the possibility of timely treatment, and the general public and scenic staff can be used at the critical moment with minimal training. China's relevant laws and regulations have clearly explained the responsibility of active rescuers, which generally concerns the managers of the scenic areas. In addition, the promotion and related experiences of AEDs in Taiwan, Hangzhou, Shenzhen, Shanghai and other provinces and cities are very useful in the allocation of AEDs to public places in the city of Beijing. It is necessary and feasible. Therefore, it is suggested that AEDs be allocated to public places, and staff-related training should be strengthened to gradually promote the allocation and use of AED. This includes the construction of first aid packages and AED information positioning systems in public places, the sharing of the volunteer rescue networks and 120 first aid information networks to find the nearest AED position in time for emergency patients, and the further improvement and standardization of emergency medical and rescue equipment available in public places in Beijing.

\subsubsection{The Management of Medical Equipment}

Experts say that the purchase of drugs by individual scenic areas should be avoided. Because the financial income of the scenic spots is different, the burden would be too heavy if all of the related expenses were borne by the units or individuals. The experts suggest that the third-party emergency service companies should be entrusted with the form of government purchase to carry out the allocation of medical equipment and be responsible for the maintenance and renewal of first-aid drugs, including the disposal of waste. Scenic areas can coordinate with the service companies according to their actual needs and adjust the contents of the configuration and medical devices. In this way, the government can not only reduce the waste of health resources but also ensure the scientific nature of first aid work. Because it would be funded by the government, this approach can avoid the waste of health resources caused by personal purchase. At the same time, the realization of unified management is easier with the organization and systemization of emergency service companies. The Health Planning Commission, as the leading body, regularly inspects and supervises the work of emergency service companies to form a supervision network.

Moreover, the relevant staff, who responsible for the use and management of emergency equipment, need to obtain the certificate of the first aid training center. The public who uses the medical equipment for first aid is also required to have the corresponding qualifications. The experts suggest that the rescuer should use a special record table for registration after the event and verify the professional qualification of the user to safeguard the rescue and the legitimate rights and interests of both the first aid responder and the patient. The experts also believe that the medical equipment (including AED) in the scenic area should be reported regularly after use, and the report information should be collected regularly by the 120 first aid center of the city so that the information on the frequency of emergency types of the city is easy to obtain and the improvement of the first aid work in the scenic area can be seen. Additionally, it would be helpful to promote the location 
of AED sites in the scenic areas and announce them to tourists or rescue volunteers in the form of a mobile APP and other convenient forms.

\section{Discussion}

\subsection{The Burden of Purchasing Drugs}

Under the premise of unified allocation of prehospital drugs, the purchase channel and cost are two of the important issues that need clarification. Because of the different scale and management characteristics of the scenic areas at all levels, it may be difficult to achieve unity of the configuration if first aid drugs are purchased by individual sites, and it would be easy to develop problems with uneven quality and difficulty with supervision. In the demonstration meeting, the experts have proposed that, if government capital is invested, this process can be unified with a government purchase order for the equipment required by the related manufacturers and enterprises to purchase the medical equipment needed to achieve the unity of model, quality and channel and to avoid increasing the burden on some public places with limited income.

\subsection{The Feasibility of the Participation of Third-Party Companies}

Learning to form a relatively perfect management system is the key to ensuring the normal operation of emergency work in the scenic area setting. Because there are great differences between the scenic area and the professional medical institutions themselves, there are some difficulties in the maintenance and renewal of the emergency medical equipment. It is more appropriate to ensure that the professional standards are difficult to be realized. First, as a professional organization, the emergency service company has conducted special research on the allocation of emergency technology and equipment in public places. It has sufficient professional background support, and the same company is responsible for the whole process of purchase and maintenance to maintain the whole process, making it convenient for supervision. If the Health Planning Commission can transfer the implementation of the specific work, the three-level supervision and management system of "health planning department - professional emergency service company - scenic area" is formed, and social capital is introduced to be responsible for the allocation, maintenance and renewal of first-aid drugs. The scenic areas are responsible for ensuring the available of first aid materials and staff at the units. Training and usage standards are strictly implemented in accordance with relevant regulations. This can save health resources to the maximum extent and ensure that the measures of the management approach can be implemented. Therefore, it is suggested that the above contents be clearly defined in the specific description of the management approach.

\subsection{The Necessity of Delivering Information}

For prehospital emergency allocation in public places, the corresponding medical equipment, which differs according to the characteristics of the patients in each place, can ensure the scientific and economic nature of the allocation of resources. At present, there are no unified emergency information records and reporting regulations for various public places in Beijing. However, in our field investigation, the records of first aid activities in some public places are spontaneous and lack fixed format and filing requirements, which is not convenient for the preservation of data and affects the use of information statistics for prehospital first-aid drugs. In the prehospital first aid research of the Mount Huangshan scenic area by Pan Haibo and others, the researchers analyzed the medical records of all the emergency patients in the scenic area for the years 2005-2010, and ultimately described the characteristics and emergency needs of the patients in the mountain scenic area. The authors put forward corresponding first aid configuration suggestions, which had strict logic and scientific nature. If the medical equipment, first aid activities, patient information and other records of the incidents in the public places in Beijing are required and standardized, the collected information will be the most valuable data for prehospital first aid and the disposition of drugs. Therefore, in the compilation of this management method and the relevant recommendations made by the experts, it is suggested that the city's 120 first aid centers use the existing first aid network to collect and analyze the prehospital first aid information from the public places in the city and strictly require the public places to complete a record of all prehospital first-aid drugs use.

\section{Conclusion}

The research results show that the overall framework of emergency medical equipment in Beijing scenic areas is relatively good and has a strong emergency mechanism. However, there is no unified standard regarding the specific contents of the medical equipment. Some of the first-aid equipment deployed by some scenic areas are obsolete and are still in the configuration of the conventional first-aid equipment (such as cutting knife, gauze, bandage, etc.). There are many scenic areas that do not know the allocation of first-aid equipment and medicines in their own units. Therefore, it is necessary to establish a unified standard for the configuration of emergency medical equipment in public places and other public places, and it is very necessary to carry out standardized management.

\subsection{Configuration and Content of Medical Equipment}

First aid kits are necessary in scenic areas because they are convenient to carry and store and are advantageous for the unified standardization of first aid in public places. It is suggested that first aid kits in scenic areas should be arranged in the form of a first aid package or first aid box, and a unified use log should be established, especially in order to manage the medical equipment. Which drugs should be used by the public and which medical devices can be used by 
professionals? The purpose of first aid packages should be to meet the first aid needs of trauma and cardiogenic diseases and grow according to actual needs. It is suggested that basic equipment, such as tweezers, scissors, gloves, thermal insulation blankets and respirators, should be deployed in all scenic areas. Respirators are made of activated carbon masks, which provide better protection for users. The configuration of disinfectant and disinfectant wipes can best meet basic bactericidal functions. Heat preservation blankets can maintain a patient's temperature well and can also be used to help construct a stretcher. Compared with the cotton quilts and other similar materials, it has better economic and storage convenience. In regard to bandages, it is suggested that elastic bandages for hemostasis and pressure bandages be considered. These two types can not only address serious injuries, such as massive bleeding, but also reduce the requirements for specific professional dressing techniques. For neck support, the staff of scenic areas can choose a simple support for both practical and economic reasons.

Commonly used drugs are recommended to serve as disinfectants; alcohol and iodophor tablets can be used for the disinfection of wounds and instruments. In addition, it is recommended that aspirin, quick-acting heart reliever and nitroglycerin be used for trauma pain relief and heart disease rescues. Other auxiliary tools include waste bags, fluorescent sticks, whistles and emergency lighting tools. Waste bags can be used for the treatment of medical waste and can also be used to collect the scattered objects of the injured. The fluorescent rods and whistles can protect the injured, evacuate the crowd and help the emergency workers find the patient's position. It is especially suitable for scenic areas with large traffic volume and dense crowds. The above medical equipment should be the basic equipment of the first-aid kit, and actual kits for each scenic spot can be increased according to the actual work needs, but it should not be lower than this standard. In addition, for the popular scenic areas in Beijing, it is suggested that an AED should also be configured. Scenic site staff should obtain CPR knowledge and skills training to protect emergency patients' lives and health and to further improve the development of prehospital emergency work in Beijing.

\subsection{Support for Professional Emergency Workers}

The public's prehospital first aid ability is limited; therefore, the emergency ambulance and professional medical staff are key. Therefore, when setting up the configuration directory, a class of medical equipment for professional personnel should be equipped to prepare for the rare circumstances. Because there may be professional medical staff in public places, they can help sites to carry out effective first-responder treatment and can also provide certain rescue work support when the professional emergency personnel arrive after the event.

\subsection{Content of the Management Approach}

The contents of the management methods can be divided into the contents of purchase and maintenance, personnel and training, supervision and management, and encouraging policies. The health administration department is responsible for the accreditation of first aid, regular supervision and inspection, and the record of the AED locations. It is suggested that the third-party first aid service is responsible for the procurement and allocation of first-aid drugs and the daily equipment maintenance and kit update work. The assigned emergency package should be managed and used by the special personnel in the landscape area. The relevant staff need to obtain the first aid qualification through a regular training process. The scenic area management department can also organize regular staff to perform emergency exercises to ensure that workers are familiar with the operation of medical equipment and the processing flow under emergency situations. In addition, after the use of the equipment, users should record data regularly. The management method should also stipulate the contents of first-aid training, qualification authentication as well as the unity of training institutions, clothing and identification, and personnel retraining time. In addition, some more important content should be clearly and properly detailed, including the scope of public places, the scope of first-aid drugs, and other important content.

\section{Acknowledgements}

The authors would like to acknowledge the staff of the Beijing Tourism Bureau for their support of the on-the-spot investigation and express the sincere gratitude to the staff involved in the interviews of this research.

\section{References}

[1] China's State Council. Regulations on the management of public places hygiene [Z]. 1987.

[2] Xiukun Wu., The urgent need of prehospital medical first aid, the necessity and urgency of formulating the "measures for the management of prehospital first aid". Chinese emergency medicine. 2010.30 (12).

[3] Baiyan Wei. Practical prehospital emergency [D]. Xi'an Jiao Tong University press.2010.

[4] Chunlin Han, Hui Yang. Emergency care [D]. People's health press.2007.

[5] Taiwan Ministry of health and welfare. Measures for the management of necessary emergency rescue facilities in public places [Z]. 2013-07.

[6] Manchester Metropolitan University, First aid handbook, [Z]. 2015-08.

[7] Jinhua central hospital of Zhejiang province. Emergency instruments and equipment management measures [Z]. 2014-11.

[8] British Red Cross First aid kit best practice: https://www.redcrossfirstaidtraining.co.uk/News-and-legislati on/latest-news/2011/August/First-aid-kit-best-practice.aspx 
[9] EPHTI, first aid management and accident prevention, [Z]. 2004-11.

[10] Yuxue Guo, Guiming Teng, Wang Shiwen. Emergency management science [M]. Lanzhou: Gansu science and Technology Press, 2009:166-173

[11] Yue Hou, $\mathrm{Lu}$ Fu. Management experience of Kunming Wujiaba Airport medical emergency equipment [J]. Chinese and foreign health digest, 2012, 9 (25): 413

[12] Haibo Pan, Xiaochun Wang, XueJun Dong, et al. Epidemiological investigation of out of hospital emergency in Mount Huangshan Mountain Scenic Area [J]. School health in China. 2011.6 (12): 1036-1037.

[13] Wei Teng. Emergency analysis and Countermeasures for sick passengers in special public hospitals in airports [J]. Chinese medicine guide, 2014, (04): 78.

[14] Xiaoyan Cao. Baiyun Airport pre-hospital first aid 3627 cases of disease spectrum analysis [J]. clinical medical engineering, 2012, (09): 1587-1588.

[15] Kunze-Szikszay, N, Krack, LA, Wildenauer, P, et al. The pre-hospital administration of tranexamic acid to patients with multiple injuries and its effects on rotational thermoelectrometry: a prospective observational study in pre-hospital emergency medicine. Scandinavian journal of trauma resuscitation \& emergency medicine. 2016.24.

[16] Baker, S P; O'Neill, B; Haddon, W Jr; Long, W B. The injury severity score: a method for describing patients with multiple injuries and evaluating emergency care. The Journal of trauma. 1974. 14 (3):187-96.

[17] Hongqiang Yang, Lihua Sun. 83 cases of emergency medical rescue in scenic spot [J]. Chinese rural medicine. 2015.19.

[18] Zengming Liang, Shengshun Qi, Zhang Jun. Analysis of 2992 cases of first aid outside hospital [J]. Chinese Journal of emergency resuscitation and disaster medicine, 2007, 2 (12): 741-757.

[19] Zaiqi Zhang, Luo Futian, Chen Bing, et al. Epidemiological investigation and analysis of pre-hospital deaths in large and medium cities of China $[\mathrm{J}] . \mathrm{N}^{\wedge} \$$ (ew medicine, 2010, 41 (11): 708-711770.

[20] Zonghao Li, Fangyi Qian. Chinese cardiopulmonary resuscitation (CPR) technology urgently needs modernization, standardization and legalization of the $[\mathrm{J}]$. China emergency resuscitation and disaster medicine journal, 2009, 4 (6): 353-355.

[21] Geng Feng. Some problems to be solved in pre-hospital resuscitation study $[\mathrm{J}]$. Chinese Journal of general practitioners, 2006, 5 (9): 54. 